\title{
1. Sonoporation at a low mechanical index
}

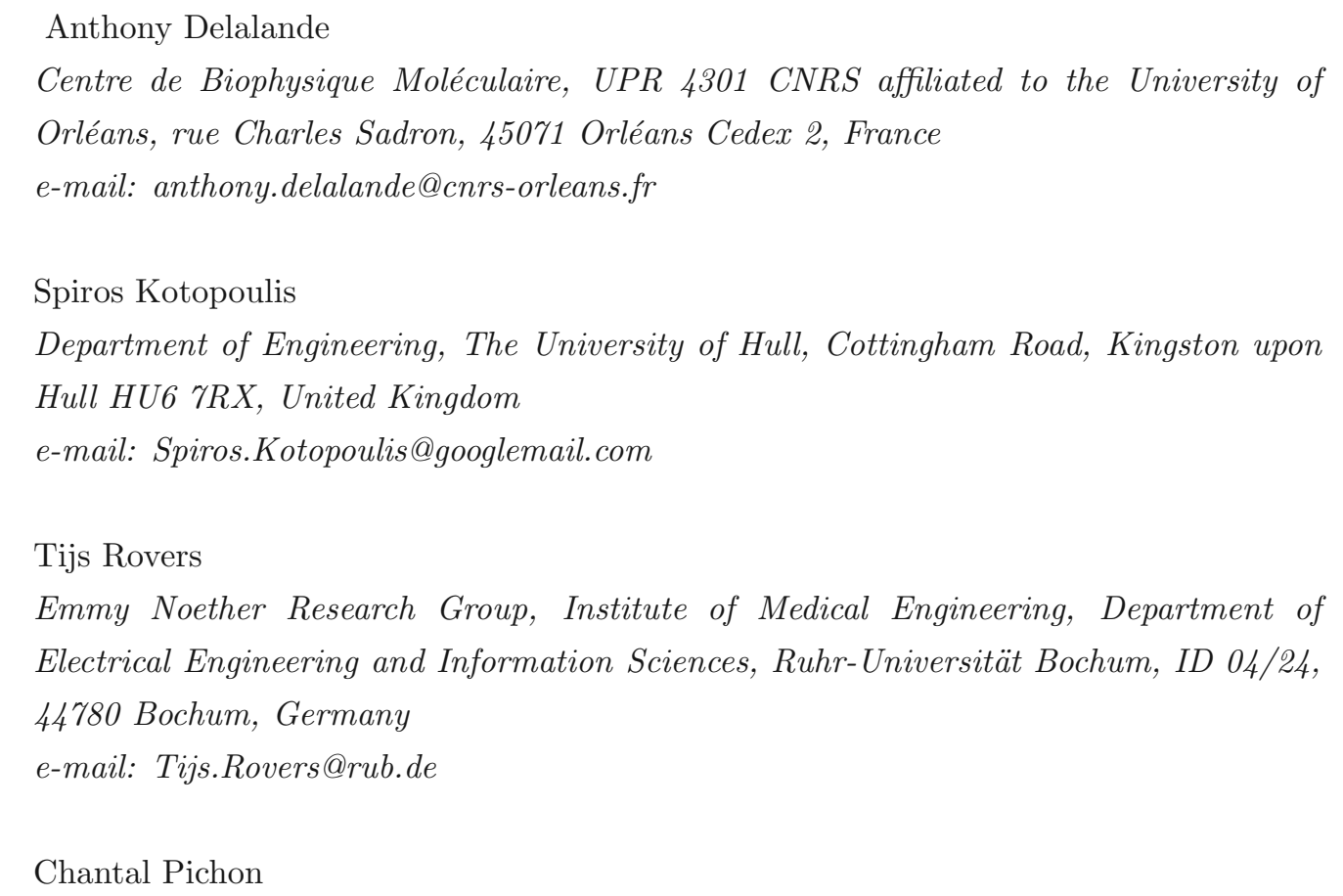




\section{Abstract}

40 Purpose: The purpose of this study was to investigate the physical mechanisms of 41 sonoporation, in order to understand and improve ultrasound-assisted drug and gene delivery. Sonoporation is the transient permeabilisation and resealing of a cell membrane with the help of ultrasound and/or an ultrasound contrast agent, allowing for the

44 trans-membrane delivery and cellular uptake of macromolecules between $10 \mathrm{kDa}$ and

$453 \mathrm{MDa}$.

46 Methods: We studied the behaviour of ultrasound contrast agent microbubbles near cancer cells at low acoustic amplitudes. After administering an ultrasound contrast

48 agent, HeLa cells were subjected to $6.6-\mathrm{MHz}$ ultrasound with a mechanical index of 0.2

49 and observed with a high-speed camera.

Results: Microbubbles were seen to enter cells and rapidly dissolve. The quick dissolution after entering suggests that the microbubbles lose (part of) their shell whilst entering.

Conclusions: We have demonstrated that lipid-shelled microbubbles can be forced to enter cells at a low mechanical index. Hence, if a therapeutic agent is added to the shell of the bubble or inside the bubble, ultrasound-guided delivery could be facilitated at diagnostic settings. In addition, these results may have implications for the safety 
Sonoporation is the transient permeabilisation and resealing of a cell membrane with the help of ultrasound and/or an ultrasound contrast agent, allowing for the trans-membrane delivery and cellular uptake of macromolecules between $10 \mathrm{kDa}$ and 3 MDa. ${ }^{1}$ Many studies have demonstrated increased drug and gene uptake of sites under sonication. $^{2-9}$ These studies presumed, that a physical membrane disruption mechanism, i.e., sonoporation, caused the increased uptake, as opposed to naturally occurring active uptake processes, such as endocytosis, that are controlled by the system biology. ${ }^{2-9}$ Although mechanical disruption with the aid of ultrasound has been attributed to violent side effects of inertial cavitation and microbubble fragmentation, most notably, the increased uptake has also been observed at low acoustic amplitudes, i.e., in acoustic regimes where inertial cavitation and microbubble fragmentation are not to be expected. An ultrasound contrast agent microbubble might act as a vehicle to carry a drug or gene load to a perfused region of interest. If the same ultrasound field that has been implicated in the sonoporation process can cause release of the therapeutic load, this load could be delivered into cells. Apart from plainly mixing ultrasound contrast agents with therapeutic agents, several schemes have been proposed to incorporate therapeutic loads to microbubbles. These include loads to the microbubble shell, ${ }^{10}$ therapeutic gases inside the microbubble, ${ }^{11}$ gas-filled lipospheres containing drugs, ${ }^{12}$ and drug-filled antibubbles. ${ }^{13}$ To understand and ameliorate ultrasound-assisted drug and gene delivery, the physics of controlled release and of sonoporation have been under investigation. That objective also forms the focus for this paper. Moreover, we studied the behaviour of ultrasound contrast agent microbubbles near cancer cells deliberately at low acoustic amplitudes in in order to probe whether sonoporation in this regime was possible; and if so, to ascertain what the microscopic mechanism might entail; and finally, to assess and scrutinise the safety aspects of ultrasound exposure in this regime.

\section{Mechanical index}

The mechanical index (MI) gives an indication of mechanical damage of tissue due to inertial cavitation. It is defined by:

$$
\mathrm{MI}=\frac{p^{-}}{\sqrt{f_{\mathrm{c}}}}
$$

where $p^{-}$is the maximum value of peak negative pressure anywhere in the ultrasound field, measured in water but reduced by an attenuation factor equal to that which would be produced by a medium having an attenuation coefficient of $0.3 \mathrm{~dB} \mathrm{~cm}^{-1} \mathrm{MHz}^{-1}$, normalised by $1 \mathrm{MPa}$, and $f_{\mathrm{c}}$ is the centre frequency of the ultrasound normalised by $1 \mathrm{MHz}$. For $\mathrm{MI}<0.3$, the acoustic amplitude is considered low. For $0.3>\mathrm{MI}>0.7$, there is a possibility of minor damage to neonatal lung or intestine. ${ }^{14}$ These are considered moderate acoustic amplitudes. For MI $>0.7$, there is a risk of cavitation if an ultrasound 
contrast agent containing gas microspheres is being used, and there is a theoretical risk of cavitation without the presence of ultrasound contrast agents. ${ }^{15}$ The risk increases with MI values above this threshold. These are considered high acoustic amplitudes. On commercial scanners, the MI has been limited to 1.9 for medical imaging. ${ }^{16}$ At low MI, microbubbles pulsate linearly, whereas at high MI, their greater expansion phase is followed by a violent collapse. During the collapse phase, when the kinetic energy of the bubble surpasses its surface energy, a bubble may fragment into a number of smaller bubbles. Fragmentation has been exclusively observed with contrast agents with thin, elastic shells. Fragmentation is the dominant disruption mechanism for these bubbles. ${ }^{17}$ Although the fragmentation of therapeutic load-bearing microbubbles must release their loads, the actual drug or gene delivery is in this case a passive process, dependent on diffusion rate and proximity to the target cells. Fragmenting microbubbles may not create pores in cells, since fragmentation costs energy. However, if a microbubble collapses near a free or a solid boundary, the retardation of the liquid near the boundary may cause an asymmetry. This asymmetry causes differences in acceleration on the bubble surface. During further collapse, a funnel-shaped jet may protrude through the microbubble, shooting liquid to the boundary. ${ }^{18}$ The pore size created by a jet has been empirically related to the microbubble expansion. ${ }^{19}$ If jets could be directed to cell layers, in case of a microbubble carrying a therapeutic load, the load could be delivered into cells. The jet formation is effected by the cavitation topology, synergistically interacting with local fluid dynamics arising through the bubble's expansion and contraction due to the ultrasound field. However, as the fluid forming the microjet is just the bulk fluid which carries no therapeutic agent, then there is no guarantee that, even with the formation of a sonopore due to jet impact with the cell membrane, therapeutic agent will enter the cell. It needs to be dislodged and mobilised from the bubble first. Furthermore, jetting has not been observed at low or moderate $\mathrm{MI},{ }^{20}$ so that fragmentation is likely to occur before any delivery takes place. By pushing the loaded microbubbles towards the vessel wall using primary radiation forces ${ }^{21}$ release can take place closer to target vessels. In a recent study, Caskey et al. pushed bubbles into tissue-mimicking gels at $\mathrm{MI}=1.5 .^{22}$ We previously studied how microclusters consisting of lipid-encapsulated microbubbles can be formed using primary and secondary radiation forces, and how these clusters can be pushed towards vessel walls. ${ }^{23}$ We found that, even at $\mathrm{MI}<0.15$, microbubble clusters can be formed and pushed within seconds.

\section{Sonoporation}

There are five non-exclusive hypotheses for explaining the sonoporation phenomenon. These have been summarised in Figure 1: push, pull, jetting, shear, and translation. ${ }^{24}$ It has been hypothesised that expanding microbubbles might push the cell membrane inward, and that collapsing bubbles might pull cell membranes outward. ${ }^{25}$ These 
mechanisms require microbubbles to be present in the close vicinity of cells. A separate release mechanism should then ensure localised delivery. Although jetting only occurs in a high-MI regime, it is very effective in puncturing cell membranes. Jetting has been observed through cells using ultrasound contrast agent microbubbles. However, the acoustic impedance of the solid cell substratum formed the boundary to which the jetting took place, not the cell itself. ${ }^{26}$ Also, there has not been any proof yet of cell survival after jetting. In a separate study, we excluded the role of jetting as a dominant mechanism in sonoporation. ${ }^{27}$ If a microbubble is fixed to a membrane, the fluid streaming around the oscillating bubbles creates enough shear to rupture the membrane. ${ }^{28}$ Here again, separate release mechanism should then ensure localised delivery. Finally, it has been speculated that lipid-encapsulated microbubbles, in compressed phase, translate through cell membranes or channels in the cell membrane. In case of therapeutic loading, the load would be delivered directly into the target cell. The main advantage of the latter mechanism is that microbubble translation by means of ultrasonic radiation forces requires very low acoustic pressures. Hence, and potential damaging bioeffects due to inertial cavitation can be ruled out.

\section{Materials and methods}

\section{Sonoporation configuration}

In previous studies, increased gene uptake was demonstrated at $\mathrm{MI}<0.3 .{ }^{29,30}$ We used a similar sonoporation configuration for our experiments. An overview of the experimental setup is shown in Figure 2. A signal consisting of 50 cycles with a centre frequency of $6.6 \mathrm{MHz}$ and a pulse repetition frequency of $10 \mathrm{kHz}$, i.e., a duty cycle of $7.5 \%$, was generated by an AFG 3102, dual channel arbitrary function generator (Tektronix, Inc., Beaverton, OR, USA), amplified by a 150A250 radio-frequency (RF) amplifier (Amplifier Research, Souderton, PA, USA) set to maximum gain, and fed to a custom-built $6.6-\mathrm{MHz}$ ultrasound transducer with a hexagonal lithium niobate $y-36^{\circ}$-cut active element with a maximum width of $25 \mathrm{~mm} .{ }^{31}$ The peak-negative acoustic pressure was measured to be $0.5 \mathrm{MPa}$ in a separate tank and in the sonication chamber itself. This corresponds to an MI of 0.2. The transducer was placed in a custom-built, $260 \times 160 \times 150(\mathrm{~mm})^{3}$ Perspex sonication chamber, in which an OptiCell ${ }^{\circledR}$ cell culture chamber (Nunc GmbH \& Co. KG, Langenselbold, Germany) was placed. One side of the cell culture chamber contained a monolayer of $1.6 \times 10^{6} \mathrm{HeLa}$ cells that had been cultured in MEM with Earl's salts medium (PAA Laboratories GmbH, Pasching, Austria) supplemented with $10 \% \mathrm{v} / \mathrm{v}$ heat-inactivated fetal calf serum, GlutaMAX ${ }^{\mathrm{TM}}$ (Life Technologies Gibco, Paisley, Renfrewshire, UK), $1 \% \mathrm{v} / \mathrm{v}$ of non-essential amino-acids (PAA), penicillin $\left(100\right.$ units $\left.\mathrm{ml}^{-1}\right)$ and streptomycin $\left(100 \mu \mathrm{g} \mathrm{ml}^{-1}\right)(\mathrm{PAA})$, at $37^{\circ} \mathrm{C}$ in a humidified atmosphere containing $5 \% \mathrm{CO}_{2}$. The cells were used when there was 60-80\% confluency. Ultrasound contrast agent was injected into the cell culturing 
chamber before each experiment. Several lipid-shelled ultrasound contrast agents were tested in this study. In this paper, we present results of a $3.33 \%$ dilution of MicroMarker $^{\text {TM }}$ (VisualSonics B.V., Amsterdam, Netherlands), a lipid-shelled agent with a mean diameter of $2.5 \mu \mathrm{m}$. A customised BXFM-F microscope unit with an LCAch N 20×/0.40 PhC (Olympus Deutschland GmbH, Hamburg, Germany) and a LUMPlanFL $60 \times / 0.90$ water-immersion objective (Olympus) was placed on top of the sonication chamber. The colour charge coupled device (CCD) of a PHOTRON FastCam MC-2.1 high-speed camera (VKT Video Kommunikation GmbH, Pfullingen, Germany) was connected to the microscope. The sensor was rotated to make sure that in all recorded movies, the ultrasound is directed from the left to the right of the frame.

\section{Fluorescence configuration}

An overview of the setup used for the fluorescence experiments is shown in Figure 3. It is almost identical to the setup described in the previous section. However, here, the signal consisting of 40 cycles with a centre frequency of $6.6 \mathrm{MHz}$ and a pulse repetition frequency of $10 \mathrm{kHz}$, i.e., a duty cycle of $6.1 \%$, was amplified using a 2100L, +50-dB RF amplifier (Electronics \& Innovation, Rochester, NY, USA) and fed to our custom-built 6.6-MHz ultrasound transducer. ${ }^{31}$ Prior to injection in the OptiCell ${ }^{\circledR}$, the MicroMarker ${ }^{\circledR}$ contrast agent was labelled using a DiD $\left(\operatorname{DilC}_{18}(5)\right)$ lipophilic fluorescent probe (Vybrant ${ }^{\mathrm{TM}}$ Molecular probes, Invitrogen, San Diego, CA, USA). A ratio of $1 \mu \mathrm{l}$ of DiD to $40 \mu \mathrm{l}$ MicroMarker ${ }^{\circledR}$ was homogenised by pipetting and incubating for 5 minutes at room temperature. Figure 4 shows how the DiD fluorescent probe bonded to the phospholipid. ${ }^{32}$ Emitted $\lambda=649-703 \mathrm{~nm}$ fluorescence was localised on the microbubble shell when exciting at $\lambda=633 \mathrm{~nm}$. A custom-made aluminium sonication chamber with internal dimensions of $130 \times 170 \times 35(\mathrm{~mm})^{3}$ was locked into to the $x y$-stage of a $200 \mathrm{M}$ inverted confocal microscope (Carl Zeiss AG, Oberkochen, Germany) coupled with a LSM Axiovert 510 scanning device (Carl Zeiss), using an EC Plan-Neofluar 40×/1.30 Oil DIC M27 objective (Carl Zeiss AG), with automated z-stack functionality.

The peak-negative acoustic pressure was measured at the objective's field of view and corresponded to $\mathrm{MI}=0.2$.

To ensure that the microbubbles were not naturally attracted to the cells, $30 \mu \mathrm{l}$ MicroMarker ${ }^{\circledR}$ was diluted into $700 \mu \mathrm{l}$ of distilled water and tested for electrophoretic mobility ( $\zeta$-potential) using a Zetasizer 3000 (Malvern Instruments, Malvern, Worcestershire, United Kingdom).

To measure the thickness of the cultured cells $10^{5}$ HeLa cells were seeded into a OptiCell ${ }^{\circledR}$. The cell plasma membrane was labelled with DiD lipophilic fluorescent probe (Vybrant ${ }^{\mathrm{TM}}$ Molecular probes) according to the manufacturers protocol. The membrane fluorescence was measured using a 200M confocal microscope. Cell thickness was calculated from the difference between the upper and lower slices where fluorescence was seen. 
We recorded 23 movies under $6.6-\mathrm{MHz}$ sonication at frame rates between 500 and 2000 frames per second, representing 15 minutes of real-time exposure. Of these, 11 movies were recorded using fluorescence. In addition we recorded 10 control movies, with a total duration of 22 minutes.

\section{Results and discussion}

Throughout this section, the optical $z$-axis is defined from distal-to-focus (negative) to proximal-to-focus (positive), with $z=0$ as the focal plane.

Figure 5 shows $z$-stacks of fluorescence emitted by the DiD dye attached to the membranes of four typical HeLa cells, representing the cell geometry. In total, the thicknesses of 42 cells were measured. The cultured cells were found to be $13 \pm 2 \mu \mathrm{m}$ thick. Clearly, these cells had thicknesses much greater than ultrasound contrast agent microbubble oscillations amplitudes at $\mathrm{MI}=0.2$.

We analysed our optical system and compared our results to bubbles and cells that were slightly out of focus, to rule out that the movement of the bubble takes place in a plane different from that of the cell. Figure 6 shows a $z$-stack of two ultrasound contrast agent microbubbles, similar to Figure 10 of Postema et al. ${ }^{33}$ Proximal-to-focus Airy disks can be seen around the bubbles, whereas distal-to-focus the bubble boundaries are blurred. Note that the boundary contrast is maximal just proximal-to-focus. ${ }^{33}$

At a centre frequency of $6.6 \mathrm{MHz}$, we recorded 17 events of microbubbles entering HeLa cells. After entering, the microbubbles were observed to quickly dissolve. As an example, Figure 7 shows an event resampled at $3.4 \mathrm{~Hz}$ and $40 \mathrm{~Hz}$, respectively, where two bubbles were pushed to a cell during $11 \mathrm{~s}$ of sonication. A microbubble "A" of $4-\mu \mathrm{m}$ diameter entered the cell and dissolved, whereas a microbubble "B" of $2-\mu \mathrm{m}$ diameter stuck to the cell membrane.

Figures 8 and 9 show two similar events, where fluorescence-coated microbubbles were used. The left panels show a microbubble apparently penetrating through the cell membrane in optical focus. Approximately $70 \mathrm{~ms}$ after the ultrasound has been switched on a microbubble is seen to penetrate through the cell membrane in Figure 8. In Figure 9 the microbubble is seen to penetrate through the cell membrane approximately $24 \mathrm{~ms}$ after the ultrasound has been switched on. The right panels show a $z$-stack through the entire cell, to record whether the apparent microbubble entry is actually into the cell.

For both events, Figure 10 shows average fluorescent intensities in two regions of interest, one inside the cell, and one control region. In both events, most fluorescence from apparent microbubble entry can be observed within $5 \mu \mathrm{m}$ proximal to optical focus, thus well within the cells themselves.

Figure 11 shows frames in optical focus from the events in Figures 8 and 9, before sonication and approximately 8 minutes after sonication. Clearly, fluorescence has transferred into the cells and remained inside the cells long after sonication. 
At these low acoustic amplitudes, inertial cavitation, fragmentation, and jetting should not occur. Hence, as a mechanism in sonoporation at low MI, these phenomena might justifiably be neglected.

Our observations do not explain why some microbubbles enter a cell and others don't. The quick dissolution after entering suggests that the microbubble loses (part of) its shell whilst entering.

The $\zeta$-potential measurements showed that the microbubble shells had a charge of $-43.9 \pm 2.4 \mathrm{mV}$. As cells have a natural negative charge ${ }^{34,35}$ the ultrasound contrast agent should be repelled by the cells, in our recordings we see that, once the ultrasound was turned on, the microbubbles would be attracted to the closest cell, independent of the direction of the sound field. This supports the recent finding that cell membranes can be acoustically active, ${ }^{36}$ and therefore interact with microbubbles.

Other cell types than HeLa cells must be used in follow-up studies, to investigate differences in bubble-cell interaction.

\section{Conclusions}

We have demonstrated that lipid-shelled microbubbles can be forced to enter cells at a low MI. Hence, if a therapeutic load is added to the bubble, ultrasound-guided delivery could be facilitated at diagnostic settings.

In addition, these results may have implications for the safety regulations on the use of ultrasound contrast agents for diagnostic imaging.

\section{Acknowledgements}

This work has been supported by DFG Emmy Noether Programme Grant 38355133 and EPSRC Grant EP/F037025/1. The authors are grateful to Conseil Regional for A. Delalande's fellowship.

\section{References}

1 M. Postema and O. H. Gilja, Curr. Pharm. Biotechnol., 2007, 8(6), 355-361.

2 S. Bao, B. D. Thrall and D. L. Miller, Ultrasound Med. Biol., 1997, 23, 953-959.

${ }^{3}$ S. Chen, R. V. Shohet, R. Bekeredjian, P. Frenkel and P. A. Grayburn, J. Am. Coll. Cardiol., 2003, 42(2), 301-308.

${ }^{4}$ W. J. Greenleaf, M. E. Bolander, G. Sarkar, M. B. Goldring and J. F. Greenleaf, Ultrasound Med. Biol., 1998, 24(4), 587-595. 
5 I. Kondo, K. Ohmori, A. Oshita, H. Takeuchi, S. Fuke, K. Shinomiya, T. Noma, T. Namba and M. Kohno, J. Am. Coll. Cardiol., 2004, 44(3), 644-653.

6 N. Kudo, K. Okada and K. Yamamoto, Biophys. J., 2006, 96(12), 4866-4876.

7 J. Lindner and S. Kaul, Echocardiography, 2001, 18(4), 329-337.

8 K. Tachibana, T. Uchida, K. Ogawa, N. Yamashita and K. Tamura, Lancet, 1999, 353, 1409.

9 S. Tinkov, R. Bekeredjian, G. Winter and C. Coester, J. Pharm. Sci., 2009, 98(6), 1935-1961.

10 A. L. Klibanov, Adv. Drug Delivery Rev., 1999, 37, 139-157.

11 M. Postema, A. Bouakaz, F. J. ten Cate, G. Schmitz, N. de Jong and A. van Wamel, Ultrasonics, 2006, 44(S1), e109-e113.

12 M. J. Shortencarier, P. A. Dayton, S. H. Bloch, P. A. Schumann, T. O. Matsunaga and K. W. Ferrara, IEEE Trans. Ultrason., Ferroelect., Freq. Contr., 2004, 51(7), 822-831.

13 M. Postema, F. J. ten Cate, G. Schmitz, N. de Jong and A. van Wamel, Lett. Drug Des. Discov., 2007, 4(1), 74-77.

14 British Medical Ultrasound Society: 'Guidelines for the safe use of diagnostic ultrasound equipment', 2000.

15 G. ter Haar, Med Biol Eng Comput, 2009, 47, 893-900.

16 J.-U. Voigt, Methods, 2009, 48, 92-97.

17 M. Postema and G. Schmitz, Ultrason. Sonochem., 2007, 14(4), 438-444.

18 A. Philipp and W. Lauterborn, J. Fluid Mech., 1998, 361, 75-116.

19 T. Kodama and K. Takayama, Ultrasound Med. Biol., 1998, 24(5), 723-738.

20 M. Postema, A. van Wamel, F. J. ten Cate and N. de Jong, Med. Phys., 2005, 32(12), $3707-3711$.

21 P. A. Dayton, J. S. Allen and K. W. Ferrara, J. Acoust. Soc. Am., 2002, 112(5), 2183-2192.

22 C. Caskey, S. Qin, P. A. Dayton and K. W. Ferrara, J. Acoust. Soc. Am., 2009, 125(5), EL183-EL189.

23 S. Kotopoulis and M. Postema, Ultrasonics, 2010, 50, 260-268. 
24 M. Postema, O. H. Gilja and A. van Wamel: 'Fundamentals of Medical Ultrasonics', ed. M. Postema, Spon press, London, 2011, 205-217.

25 A. van Wamel, K. Kooiman, M. Harteveld, M. Emmer, F. J. ten Cate, M. Versluis and N. de Jong, J. Control. Release, 2006, 112(2), 149-155.

26 P. Prentice, A. Cuschieri, K. Dholakia, M. Prausnitz and P. Campbell, Nature Phys., 2005, 1, 107-110.

27 M. Postema and O. H. Gilja, Biomed. Eng., 2010, 55, S19-S20.

28 P. Marmottant and S. Hilgenfeldt, Nature, 2003, 423, 153-156.

29 A. Delalande, M.-F. Bureau, P. Midoux, A. Bouakaz and C. Pichon, Ultrasonics, 2009, 50, 269-272.

30 K. Kaddur, L. Lebegue, F. Tranquart, P. Midoux, C. Pichon and A. Bouakaz, IEEE Trans. Ultrason. Ferroelectr. Freq. Control, 2010, 57(7), 1558-1567.

31 S. Kotopoulis, H. Wang, S. Cochran and M. Postema, Proc. IEEE Ultrason. Symp., 2010, accepted.

32 P. Livanec and R. Dunn, Langmuir, 2008, 24(24), 14066-14073.

33 M. Postema, A. Bouakaz, C. T. Chin and N. de Jong, IEEE Trans. Ultrason., Ferroelectr., Freq. Control, 2003, 50(5), 523-536.

34 B. Ehrenberng, V. Montana, M.-D. Wei, J. Wuskell and L. Loew, Biophys. J., 1988, 53, $785-794$.

35 A. Takahashi, H. Yamaguchi and H. Miyamoto, Am. J. Physiol., 1993, 265(2), C328-C336.

36 B. Krasovitski, V. Frenkel, S. Shoham and E. Kimmel, Proc. Nat. Acad. Sci., 2011, accepted. 


\section{List of Figures}

1 Possible mechanisms of sonoporation: a) push, b) pull, c) jetting, d) shear, e) translation. Based on Figure 9.2 in Postema et al. ${ }^{24}$. . . . .

2 Experimental setup ( $t o p)$ and a close-up of the sonoporation configuration

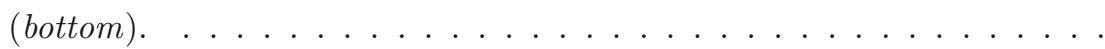

3 Experimental setup (top) and a close-up of the fluorescence configuration (bottom). . . . . . . . . . . . . . . . .

4 Schematic representation of $\mathrm{DiD}\left(\mathrm{DilC}_{18}(5)\right)$ lipophilic fluorescent probe bonding to phospholipid. ${ }^{32} \ldots \ldots \ldots$. . . . . . . . . 16

$5 z$-stacks of fluorescence emitted by the DiD dye attached to the membranes of four typical HeLa cells, representing the cell geometry. . .

$6 z$-stack of two ultrasound contrast agent microbubbles. Proximal-to-focus Airy disks can be seen around the bubbles, whereas distal-to-focus the bubble boundaries are blurred. Microbubble A has a diameter of $2 \mu \mathrm{m}$, whereas microbubble B has a diameter of $3 \mu \mathrm{m}$. Each frame corresponds to a $11 \times 11(\mu \mathrm{m})^{2}$ area. . . . . . . . . . . . . . . .

7 A sonoporation event including microbubble dissolution during $11 \mathrm{~s}$ of sonication (left) and selected frames of the microbubble entereing a cell (right). Microbubble "A" entered the cell and dissolved, whereas microbubble "B" stuck to the cell membrane. Each frame corresponds to a $23 \times 23(\mu \mathrm{m})^{2}$ area. . . . . . . . . . . . . . . . .

8 Microbubble of 5 - $\mu \mathrm{m}$ diameter apparently penetrating through the cell membrane in optical focus (left); $z$-stack through the entire cell, to record whether the apparent microbubble entry is actually into the cell (right). Areas (A) and (C) are regions of interest inside and outside the cell, respectively. Each frame corresponds to a $76 \times 76(\mu \mathrm{m})^{2}$ area. . . . . . .

9 Microbubble of $4-\mu \mathrm{m}$ diameter apparently penetrating through the cell membrane in optical focus (left); $z$-stack through the entire cell, to record whether the apparent microbubble entry is actually into the cell (right). Areas (A) and (C) are regions of interest of high fluorescence and low fluorescence, respectively, inside the cell. The white dotted lines in the upper left frame of the right panel indicates the cell membrane. Each frame corresponds to a $52 \times 52(\mu \mathrm{m})^{2}$ area. . . . . . . . . . . . .

10 Average fluorescent intensities in the regions of interest (ROI) of Figures 8 (left) and 9 (right). Bold lines represent ROI (A) inside the cells, whereas hairlines represent ROI $(\mathrm{C})$ the control regions. The dotted line represents the cell boundary contrast. Note that the cell boundary contrast is maximal just proximal-to-focus. . . . . . . . . . . . . . . . 
11 Columns (a) and (b) represent frames in optical focus from the events in Figures 8 and 9, respectively, before sonication and approximately 8 minutes after sonication. The white dotted lines in the right frames indicate the cell membrane boundary. The left frames correspond to $76 \times 76(\mu \mathrm{m})^{2}$ areas, whereas the right frames correspond to $45 \times 45(\mu \mathrm{m})^{2}$ areas. . . . . . . . . . . . . . . . . . 
(a)

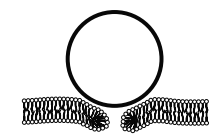

(b) $\theta$<smiles>[13CH]=[13CH][13CH3]</smiles>

(C)<smiles>c1ccccc1</smiles><smiles>O</smiles>

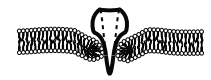

(d)
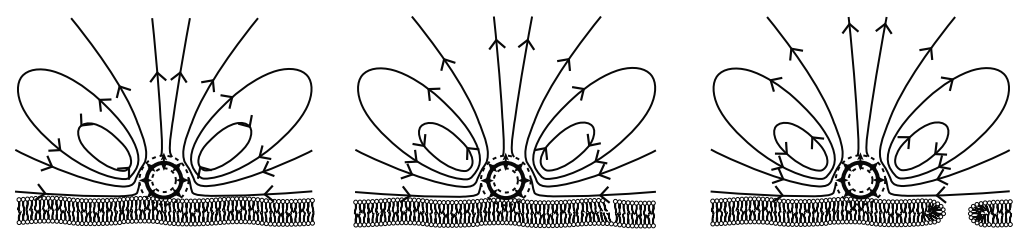

(e)
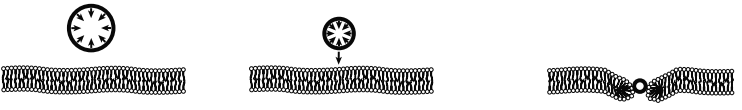

Monfom

O

Figure 1: Possible mechanisms of sonoporation: a) push, b) pull, c) jetting, d) shear, e) translation. Based on Figure 9.2 in Postema et al. ${ }^{24}$ 


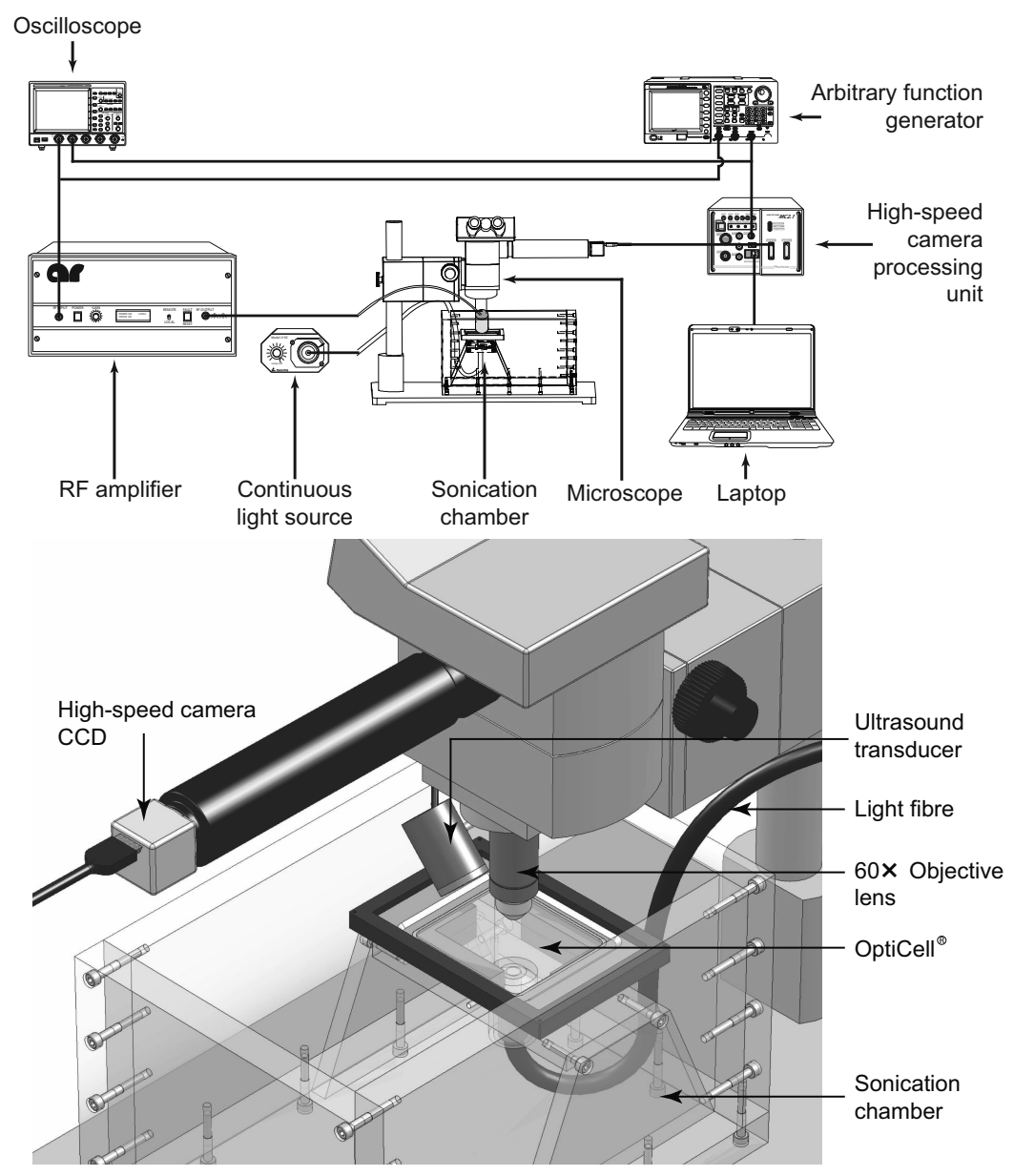

Figure 2: Experimental setup (top) and a close-up of the sonoporation configuration (bottom). 


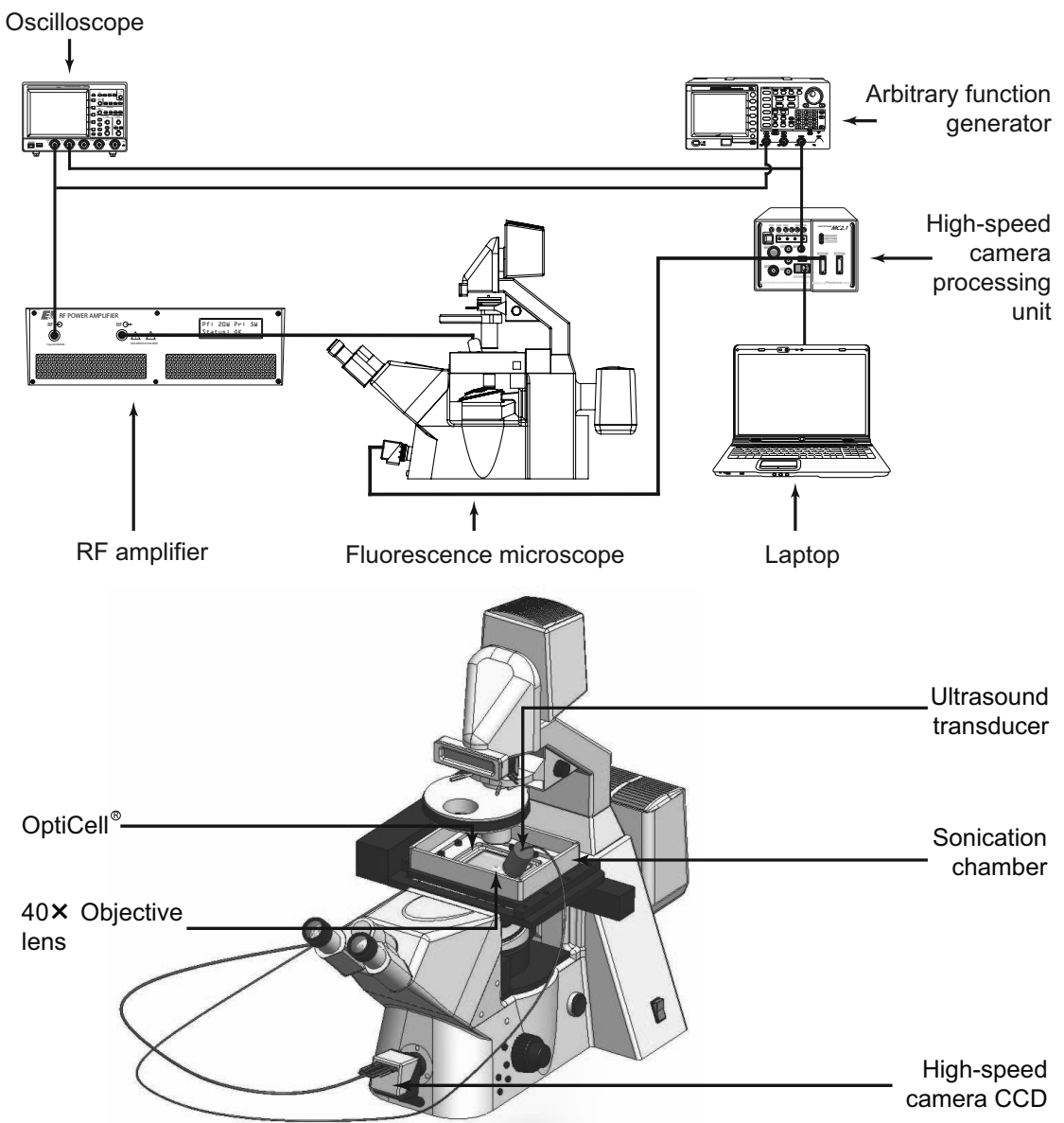

Figure 3: Experimental setup (top) and a close-up of the fluorescence configuration (bottom). 


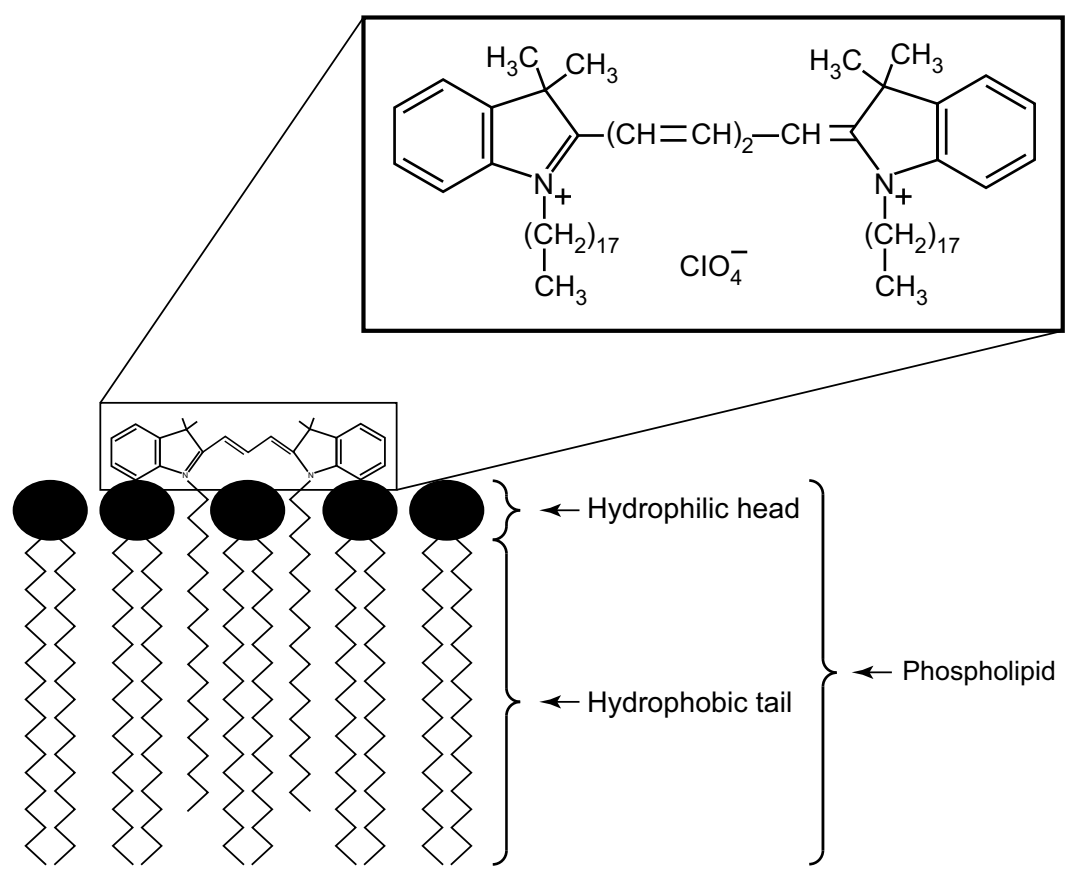

Figure 4: Schematic representation of $\mathrm{DiD}\left(\mathrm{DilC}_{18}(5)\right)$ lipophilic fluorescent probe bonding to phospholipid..$^{32}$ 

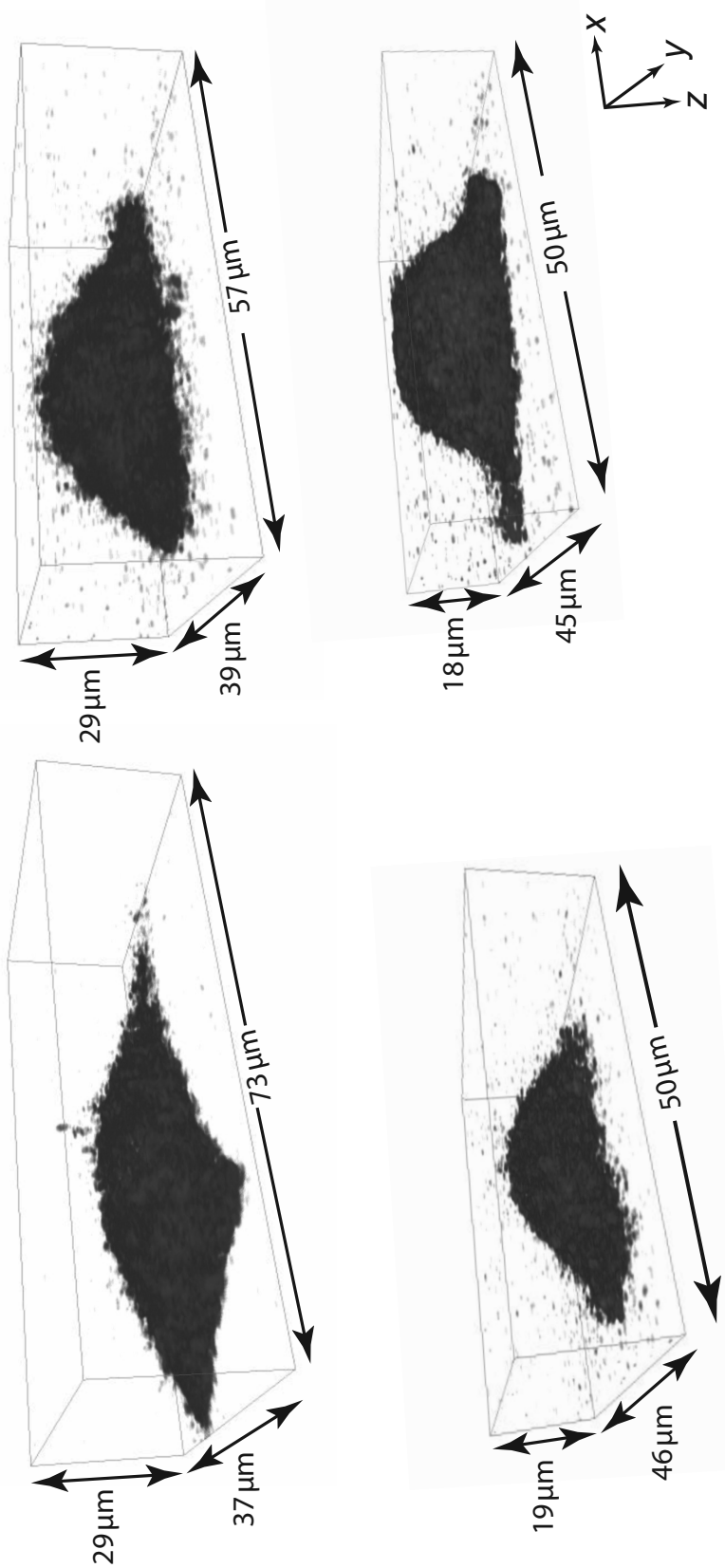

Figure 5: $z$-stacks of fluorescence emitted by the DiD dye attached to the membranes of four typical HeLa cells, representing the cell geometry. 


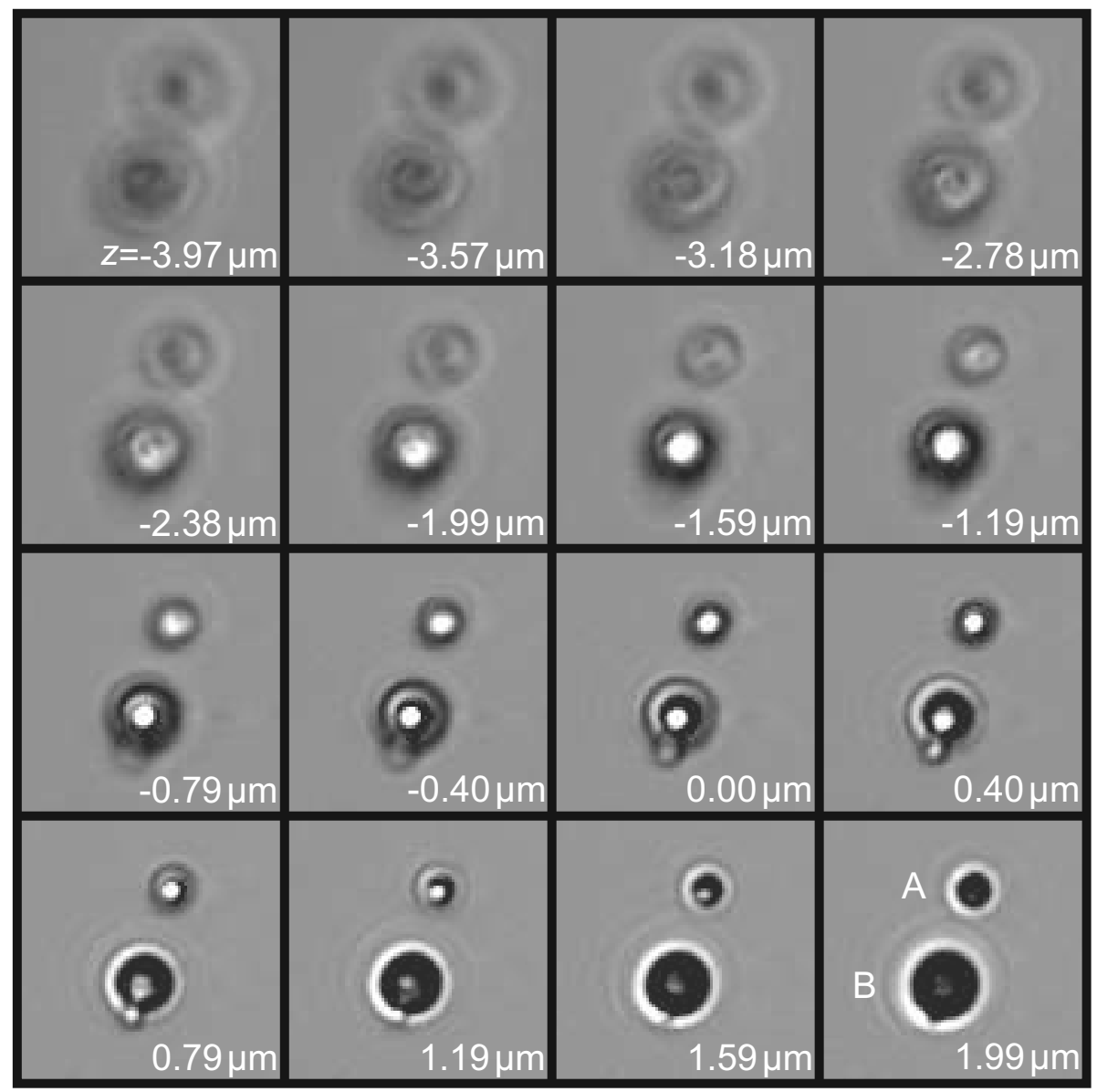

Figure 6: $z$-stack of two ultrasound contrast agent microbubbles. Proximal-to-focus Airy disks can be seen around the bubbles, whereas distal-to-focus the bubble boundaries are blurred. Microbubble A has a diameter of $2 \mu \mathrm{m}$, whereas microbubble B has a diameter of $3 \mu \mathrm{m}$. Each frame corresponds to a $11 \times 11(\mu \mathrm{m})^{2}$ area. 


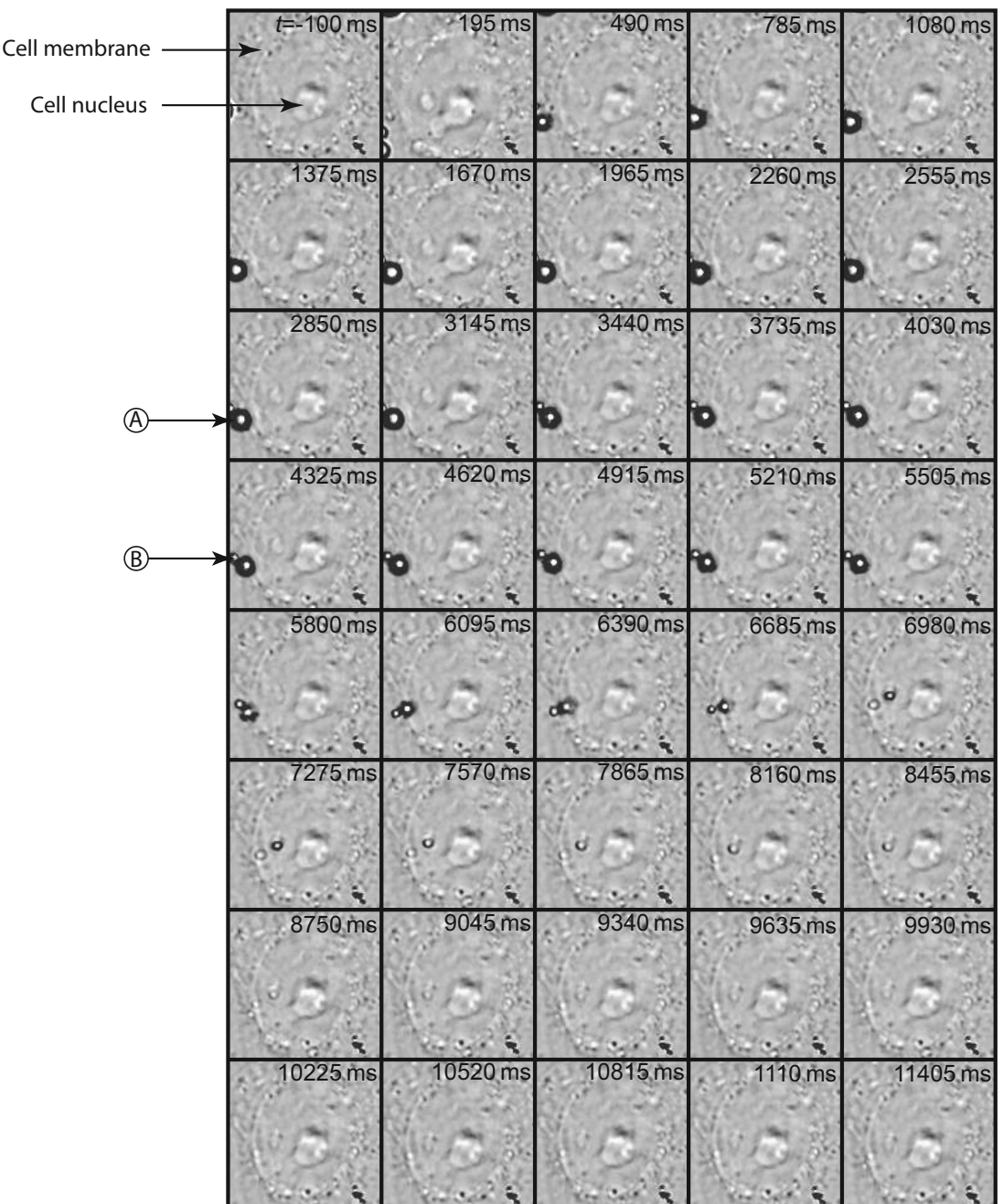

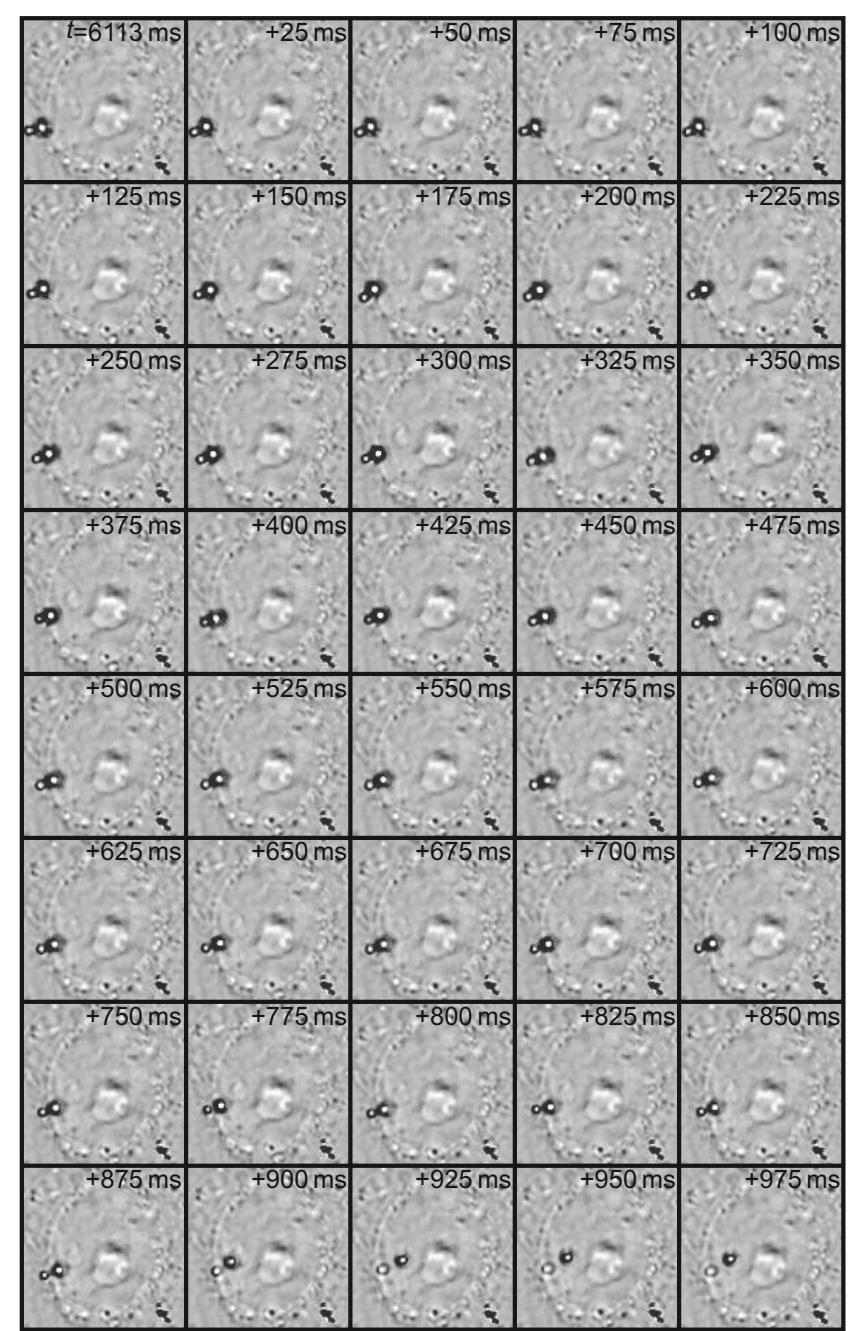



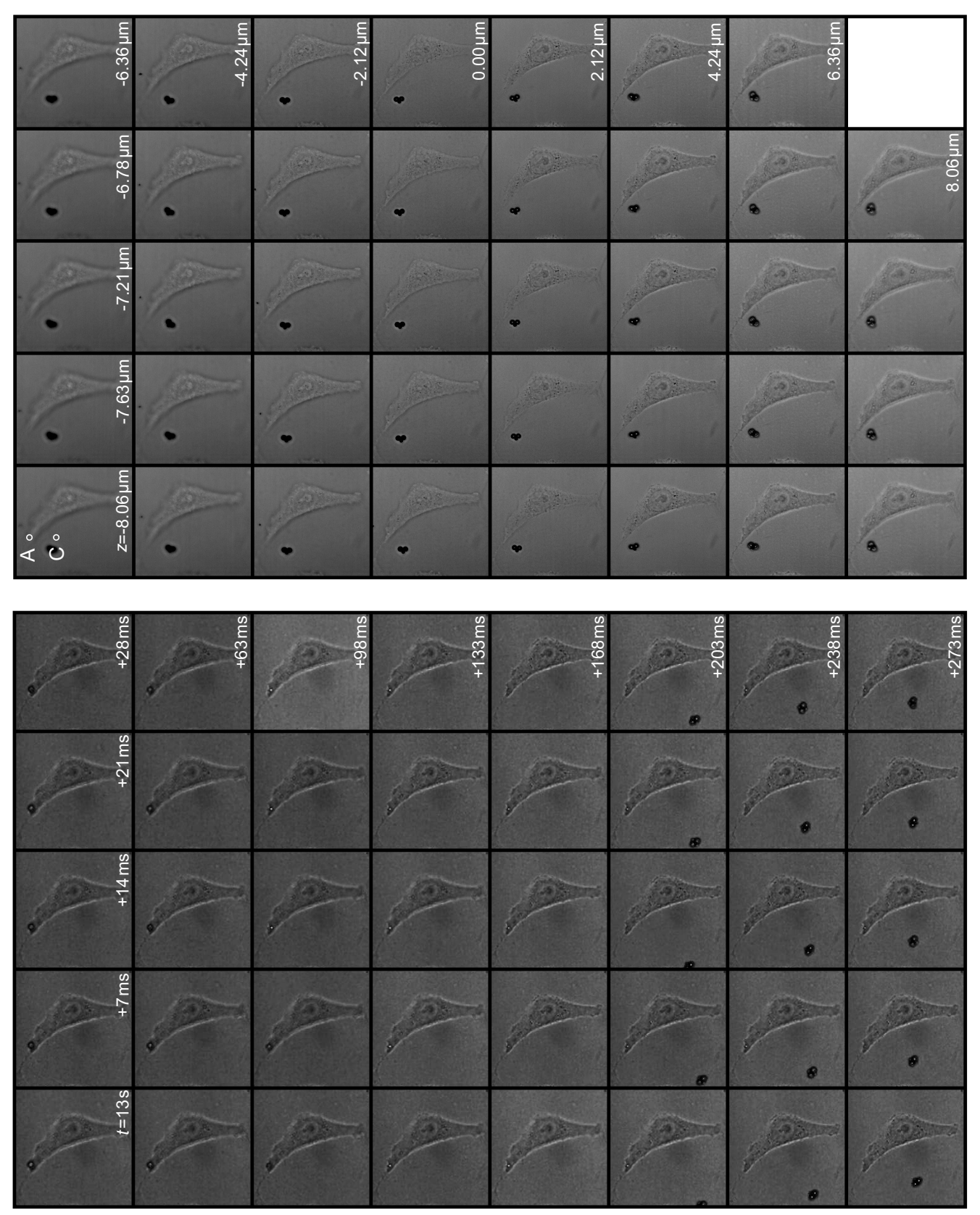

Figure 8: Microbubble of 5- $\mu \mathrm{m}$ diameter apparently penetrating through the cell membrane in optical focus (left); $z$-stack through the entire cell, to record whether the apparent microbubble entry is actually into the cell (right). Areas (A) and (C) are regions of interest inside and outside the cell, respectively. Each frame corresponds to a $76 \times 76(\mu \mathrm{m})^{2}$ area. 

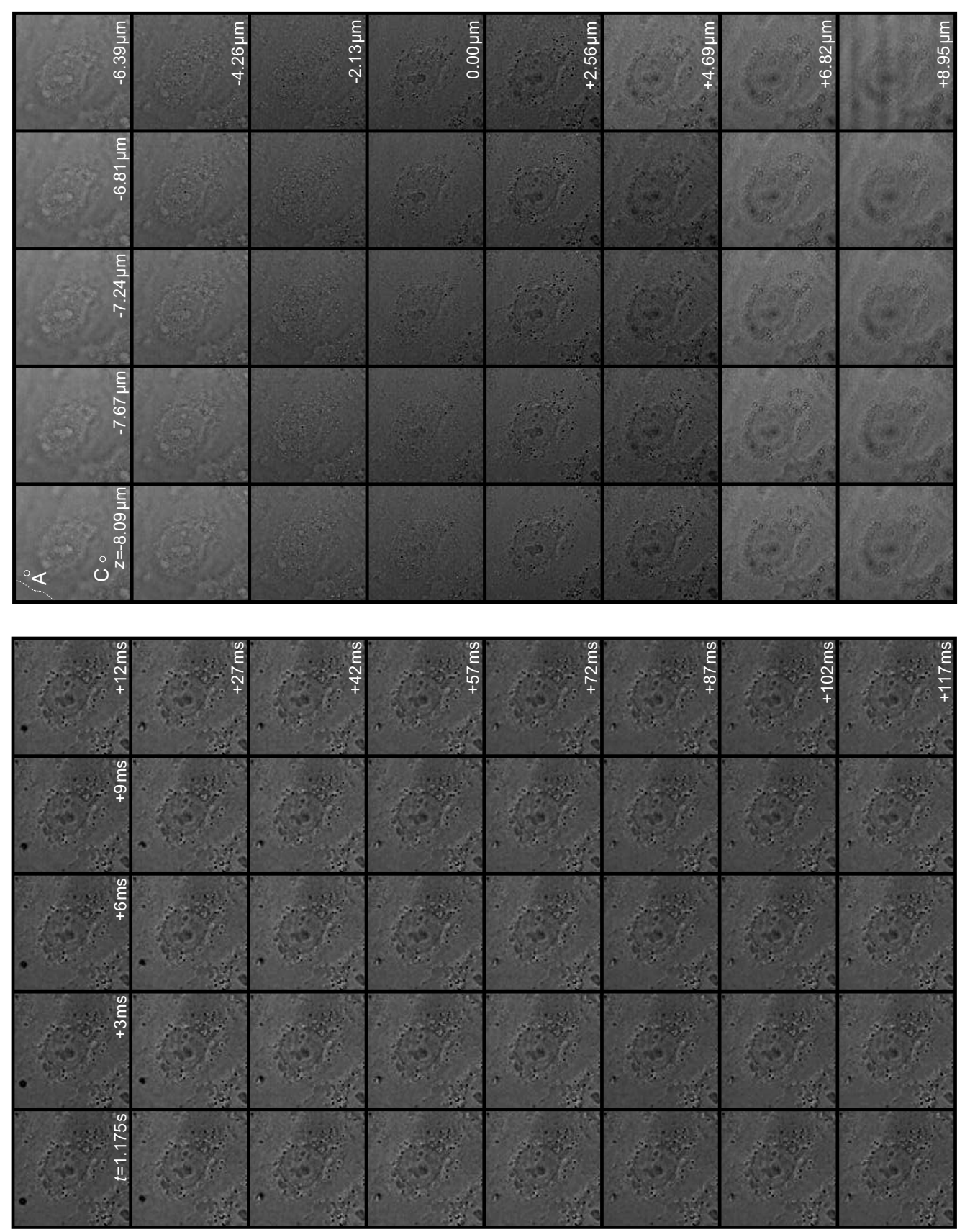

Figure 9: Microbubble of 4- $\mu \mathrm{m}$ diameter apparently penetrating through the cell membrane in optical focus (left); $z$-stack through the entire cell, to record whether the apparent microbubble entry is actually into the cell (right). Areas (A) and (C) are regions of interest of high fluorescence and low fluorescence, respectively, inside the cell. The white dotted lines in the upper left frame of the right panel indicates the cell membrane. Each frame corresponds to a $52 \times 52(\mu \mathrm{m})^{2}$ area. 


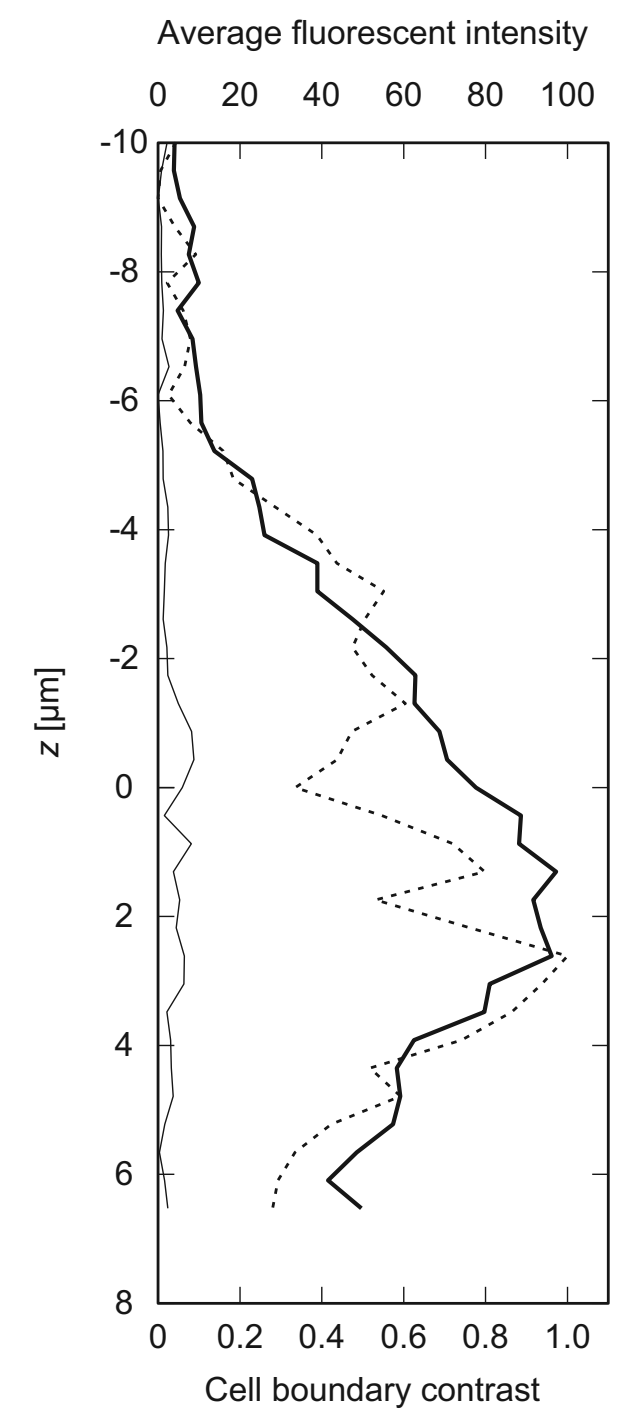

Average fluorescent intensity

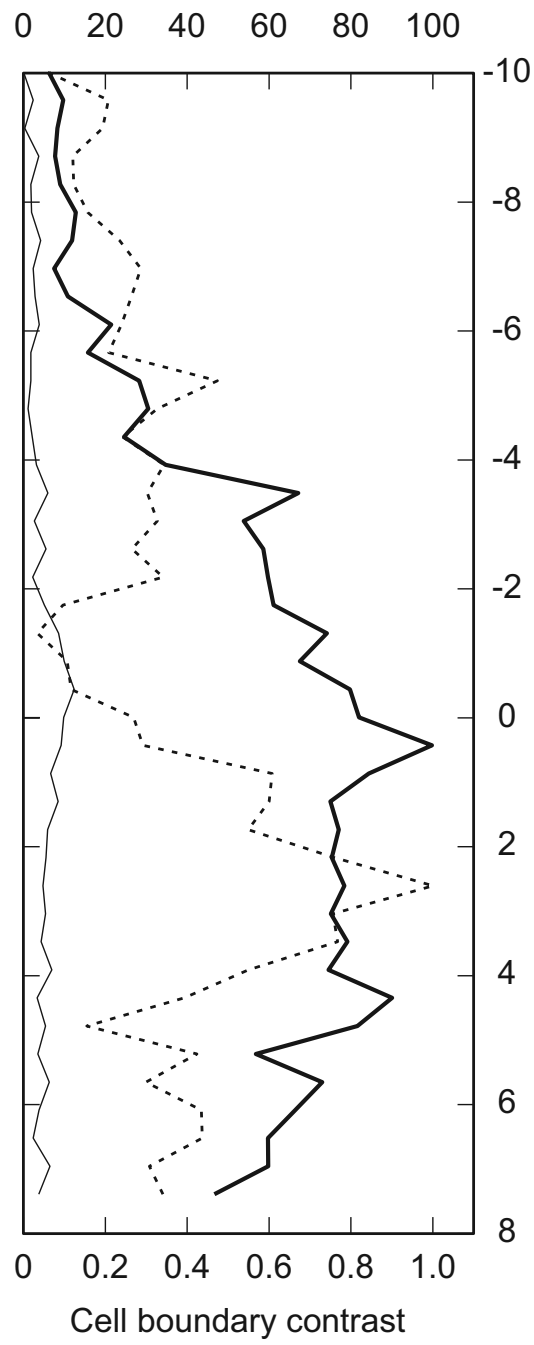

Figure 10: Average fluorescent intensities in the regions of interest (ROI) of Figures 8 (left) and 9 (right). Bold lines represent ROI (A) inside the cells, whereas hairlines represent ROI (C) the control regions. The dotted line represents the cell boundary contrast. Note that the cell boundary contrast is maximal just proximal-to-focus. 


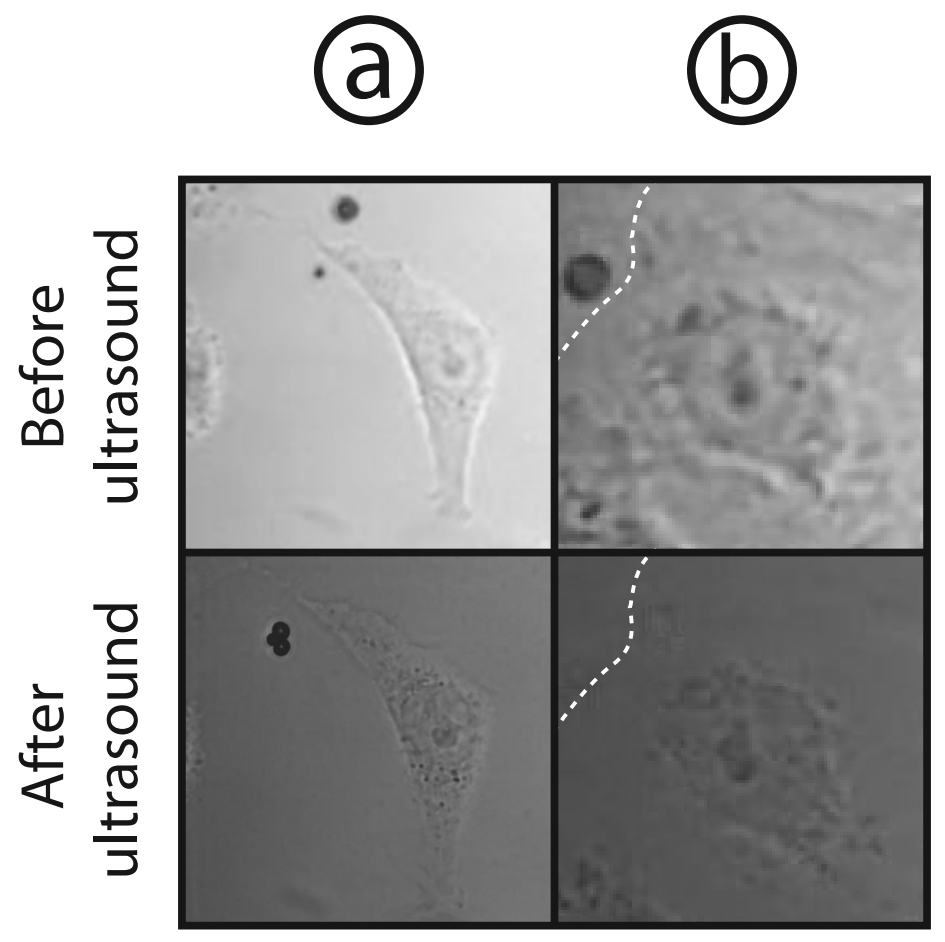

Figure 11: Columns (a) and (b) represent frames in optical focus from the events in Figures 8 and 9, respectively, before sonication and approximately 8 minutes after sonication. The white dotted lines in the right frames indicate the cell membrane boundary. The left frames correspond to $76 \times 76(\mu \mathrm{m})^{2}$ areas, whereas the right frames correspond to $45 \times 45(\mu \mathrm{m})^{2}$ areas. 